\title{
Mobile Robot Obstacle Avoidance Algorithms Based on Information Fusion of Vision and Sonar
}

\author{
Hongwei Gao ${ }^{1,2}$, Qiuyang $\mathrm{Wei}^{1}$, Yang $\mathrm{Yu}^{1}$ and Jinguo $\mathrm{Liu}^{2}$ \\ ${ }^{1}$ College of Automation and Electrical Engineering \\ Shenyang Ligong University \\ Shenyang, Liaoning, 110159, P.R. China \\ ghw1978@sohu.com \\ ${ }^{2}$ State Key Laboratory of Robotics \\ Shenyang Institute of Automation, Chinese Academy of Sciences \\ Shenyang, Liaoning, 110016, P.R. China
}

\begin{abstract}
In view of the problem of $A S-R$ autonomous wheeled mobile robot obstacle avoidance, a rapid convergence of sonar and binocular stereovision sensor distance information in order to detect and avoid obstacle algorithm is proposed in this paper. The algorithm first uses binocular camera (CCD) to get three-dimensional image of the real environment, through the stereo matching and V-disparity method which is used to calculate disparity map, then obstacles is extracted by Hough lines detection algorithm, finally we will get information about obstacles and sonar return information with $T$-S fuzzy neural network fusion, then it will output walking controlled decisions. Experimental results proved that the algorithm is effective and practical.
\end{abstract}

Keywords: Binocular stereo vision, stereo matching, $V$-disparity, obstacle detection, $T$ S fuzzy neural network

\section{Introduction}

With the development of science and technology, the application field of mobile robot is more and more widely, and had a profound impact to the human production and life. Mobile robot navigation technology is an active branch in the field and research hotspot, it is a comprehensive subject cross multidisciplinary, involved in machine vision, visual range, stereo image matching, map refactoring, video tracking technique and so on [1]. As a basic function in the navigation technology, obstacle avoidance is also an important index evaluating the degree of intelligent mobile robot, the obstacle avoidance research has become an important topic of many scholars.

Mobile robot obstacle avoidance is a robot from the starting point to the target point, using the control method to avoid related obstacles process accurately. At present, the study of robot obstacle avoidance method has also made the corresponding results. Paper [2] using the multiple ultrasonic distance sensors measuring distance and direction of the detected obstacles, after fuzzy neural network information fusion can achieve the recognition of obstacles and movement without conflict, but you can't solve the crosstalk interference between adjacent ultrasonic sensor to sweep frequency constraint problem .Paper [3] using pixel adaptive fusion method to collect visual information and normalized to the $\mathrm{A}^{*}$ algorithm for path selection, however, the algorithm has a poor realtime performance due to the complex calculation .Paper [4] describing feasible direction by adaptively adjusting the threshold of the robot, and putting forward a real-time obstacle avoidance algorithm for a given threshold to solve each threshold value corresponding feasible direction, selection of the largest threshold value corresponding to 
the direction for robot reference driving direction, but the problem of threshold sensitivity research is not enough, thus it is hard to get into practical use. Aiming at the above problems, this paper proposes a fast sensor information fusion of binocular stereo vision and sonar to real-time control the robot to avoid obstacle, which through the two CCD (Charge coupled Device) cameras to get images from different perspectives and at the same time, the image of disparity map resulting from the stereo matching, then determines the presence of obstacles and barriers in the positions of the image, and then according to its location, disparity map and camera parameters to calculate the actual distance obstacle, coupled with the distance of the sonar sensors information as T-S fuzzy neural network's input and output robot control decisions that effectively avoiding obstacles. Finally, this algorithm goes through the experimental verification.

\section{Sonar and Binocular Vision Information Fusion}

Sonar sensors, when it faces with obstacles, sonar will tap into a narrow beam of ultrasonic wave in air and ultrasound will return. According to the ultrasonic transmission time can we accurately calculate the relative distance of obstacles [5].The AS-R can install 12 sonar sensors, the angles between each sonar sensor is 30 degrees.AS-R ultrasonic distance measurement card can extend nine sonar sensors, this paper uses only 5 sonar sensors that installed in the front of the robot by default interface and other research direction have appeared. The researches on recognition technology and sketch reconstruction aspect are also not few.

Binocular vision: mainly consists of two $1 / 3$ inch SONY color CCD camera, the parameter list is as follows:

Table 1. Camera Parameter

\begin{tabular}{|c|c|}
\hline Parameter & Size \\
\hline Pixel & $640 * 480 \mathrm{px}$ \\
Frame rate & $30 \mathrm{~Hz}$ \\
Focal length & $6 \mathrm{~mm}$ \\
Power port & IEEE-1394 \\
\hline
\end{tabular}

etAccordingly, on the basis of equipped with sonar sensors, and equipped with a binocular vision system. To establish the robot coordinate system o-XcYcZc [6].Getting the range information when real-time processing of binocular vision in the robot system, then the binocular vision range information and sonar range information integration.

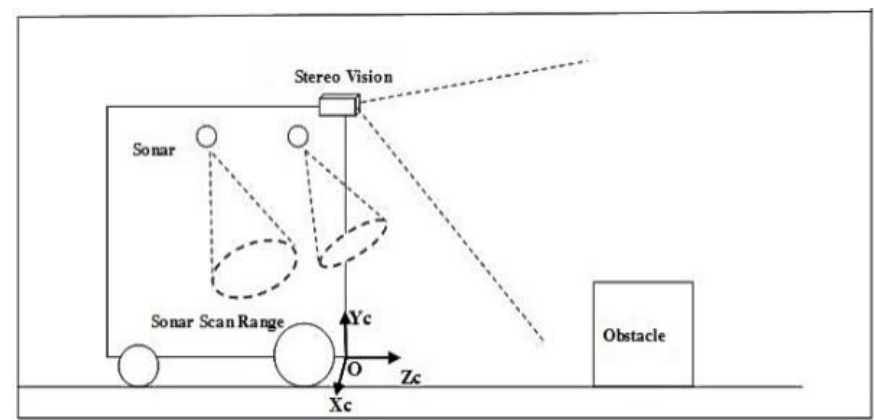

Figure 1. Sensor Integration of the AS-R Model Diagram

In this paper, using the parallel binocular stereo vision to do analysis and research. In Tao proposes a matching algorithm based on color image segmentation framework [7]. Assuming that the surface is smooth on the basis of the algorithm framework. The 
traditional algorithm of each point in the global distribution of the optimal parallax translate into each region distribution of the optimal template, in order to improve the real-time of the algorithm. In view of the binocular stereo image pairs of the camera, using the belief propagation based on image segmentation adaptive stereo matching algorithm [8-9], the algorithm is stated as in what follows:

\section{Adaptive Stereo Matching Algorithm}

\section{STEPS}

- Through regional matching algorithm to obtain the initial disparity map

- Based on robust disparity plane fitting method to extract the final disparity layer

- The belief propagation algorithm based on graph cut theory distributes and optimizes the disparity layer

One method for multidimensional fuzzy reasoning proposed by Takagi and Sugeno, which is nonlinear nature and easy to express the model of the dynamic characteristic of complex systems, called the T-S model [10].This structure has the function of local approximation and combines advantages of both fuzzy logic and neural network. Not only fuzzy and qualitative knowledge can be easily said and has good learning ability, but also has the ability of real-time processing of large amounts of data, knowledge generalization ability and structural fault tolerance, etc. Therefore, it is very suitable for fusion image and distance of both complementary information. The topology of the network is shown in Figure 2.

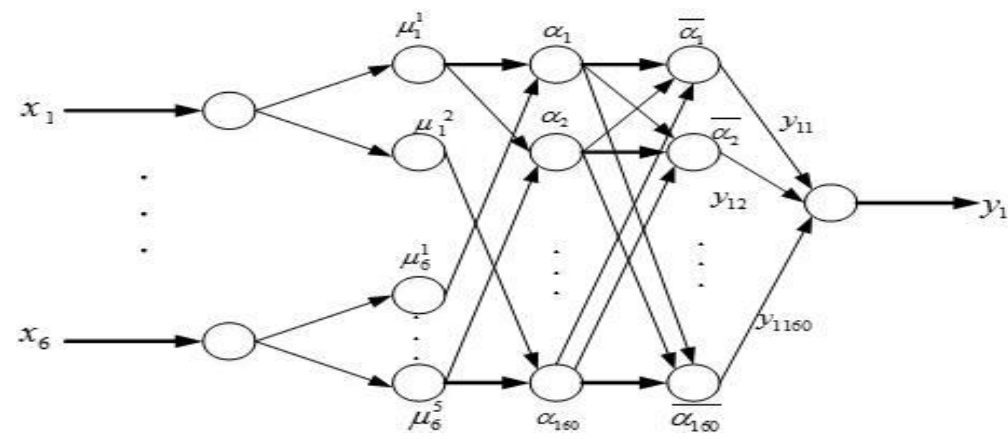

Figure 2. T-S Fuzzy Neural Network Structure

Among above, the $\mathrm{x}_{1} \sim \mathrm{x}_{6}$ is a fuzzy neural network input, the $\mathrm{x}_{1} \sim \mathrm{x}_{5}$ are obstacles' distances from obstacles to the sonar sensors detected by them, $x_{6}$ is the distance and direction angle on the location that between obstacle and the vision system, $\mathrm{y}_{1}$ outputs robot operation control angle.

\section{Obstacle Detection Algorithm Based on V-disparity Method}

As shown in the figure below. First of all, using the not calibrated binocular stereo camera to collect images, then going the step of the two-dimensional image of polar calibration in order to make the matching problem into the one-dimensional search on line. Secondly, calculating the dense disparity map and then generate the V-disparity map by using the improved Hough test fitting line algorithm to extract the straight line in Vdisparity map, then to determine the height of the obstacles and the point contacted with the ground. According to the disparity value of similar level search method to determine the width of the barrier and to lock the barrier area. Finally, calculating the distance between AS-R and obstacles. 


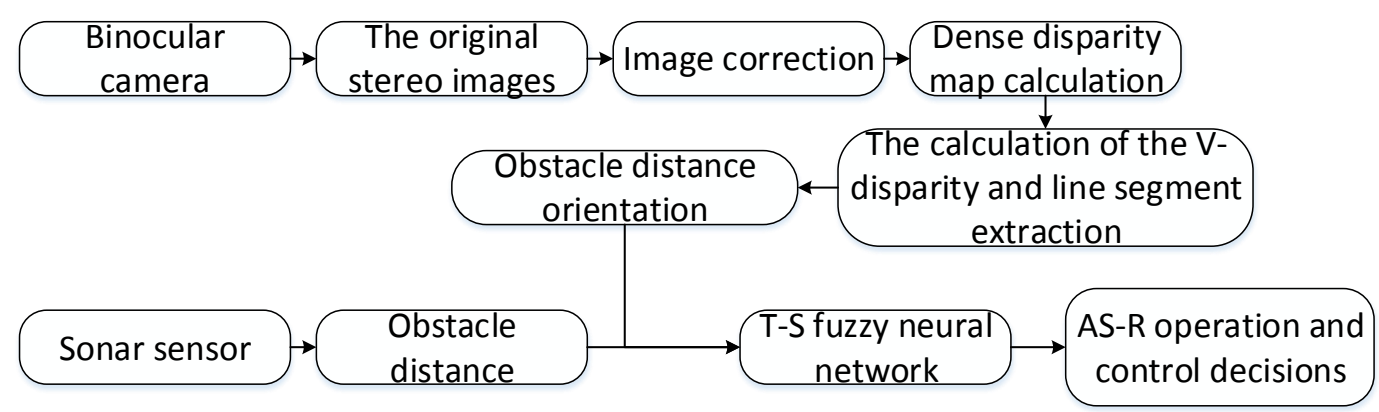

Figure 3. Based on V-disparity Method of Obstacle Avoidance Process

\subsection{Polor Correction}

Due to the actual environmental image is two-dimensional, two dimensional search is necessary from an image to find another image of the corresponding points. But two dimensional search requires large amount of calculation. Therefore, need to corrected image so that making the two images of a line parallel to the horizontal scan lines. In this paper, with no camera calibration of stereo image correction [11]. It belongs to the weak Hartley camera calibration and is a kind of high speed and precision of three-dimensional images of polar correction methods. The main steps are as follows:

3.1.1. For Plane Projection Image Correction: Set $H_{\text {and }} H^{\prime}$, respectively image plane projection transformation matrix of $I$ and $I^{\prime}, m$ and $m^{\prime}$ for corresponding points. The image of corresponding points after transformation are $\bar{m}, \bar{m}^{\prime}$ and $\bar{m}=H m, \bar{m}^{\prime}=H^{\prime} m^{\prime}$. Before correction image, the basic matrix is $F$. The outside polar equation is $m^{T} F m=0$, then adjusted the image of the poles in the horizontal direction of infinity, the fundamental matrix is

$$
\bar{F}=\left[\begin{array}{ccc}
0 & 0 & 0 \\
0 & 0 & -1 \\
0 & 1 & 0
\end{array}\right]
$$

Image after correction for outside polar equation is

$$
\left(\mathrm{H}^{\prime} \mathrm{m}^{\prime}\right)^{\mathrm{T}} \bar{F}(\mathrm{Hm})=0
$$

3.1.2. Plane Projection Transformation Matrix Calculation: With ${ }^{H_{p}}$ for the perspective projection matrix, $H_{r}$ for the rotation matrix, $H_{t}$ for vertical translation matrix and $H_{s}$ for horizontal scaling matrix.

$$
H=H_{s} H_{t} H_{r} H_{p}
$$

3.1.3. Perspective Projection, the Optimization Calculation of Rotation and Vertical Translation Matrix: Through the perspective projection, rotation and vertical translation, the very line will be in the horizontal direction and get level of correction of ideal image. So to determine the transformation matrix $\boldsymbol{H}_{t r p}$ and $\boldsymbol{H}^{{ }^{\prime}{ } \mathrm{rp}}$ is the core of the image of correction. By the corrected image with the polar equation (Equation(2)), get the evaluation function

$$
\cos t\left(\mathrm{H}_{\text {trp }}, \mathrm{H}_{\text {trp }}^{\prime}\right)=\sum_{i=1}^{N}\left[\left(\mathrm{H}_{\text {trp }}^{\prime} \mathrm{m}_{\mathrm{i}}^{\prime}\right)^{\mathrm{T}} \overline{\mathrm{F}}\left(\mathrm{H}_{\text {trp }} \mathrm{m}_{\mathrm{i}}\right)\right]^{2}
$$


Where $N$ is the image of corresponding points involved in calculating, $m i^{\prime}$ and $m i$ for homogeneous coordinates of corresponding points. Getting the transformation matrix to solve by the nonlinear least squares method.

3.1.4. Horizontal Scaling Matrix Calculation: Horizontal scaling is just to make sure that the adjusted image deformation is small, so assuming that the width of the image and height is $w$ and $h$. The four selected image edge point $a, b, c, d$, consider two straight line vertical and make the image aspect ratio constant, then $H_{s}$ is calculated.

\subsection{V-disparity Map Construction}

At the right camera coordinate system $(R C C S)$ and the left camera coordinate system (LCCS), when a line is parallel connected with the image line overlap, $v_{r}=v_{l}=v$.And to the world coordinate system of the point $P(X, Y, Z, 1)^{T}$, there is

$$
v=\frac{\left[v_{0} \sin \theta+\alpha \cos \theta\right](\mathrm{Y}+\mathrm{h})+\left[v_{0} \cos \theta-\alpha \sin \theta\right] \mathrm{Z}}{(\mathrm{Y}+\mathrm{h}) \sin \theta+\mathrm{Z} \cos \theta}
$$

Horizontal parallax at $P$ is $\Delta$ and

$$
\Delta=u_{l}-u_{r}=\frac{\alpha b}{(\mathrm{Y}+\mathrm{h}) \sin \theta+\mathrm{Z} \cos \theta}
$$

According to the picture on the right as a benchmark image of dense disparity map contained the depth of field information. Labayrade [12],etc. On the basis of the accumulation of disparity maps with the same level of parallax on each line of pixels number $N$, and $\left(\Delta \mathrm{p}, v_{\mathrm{r}}\right)$ for the new pixel coordinates. For $N$ corresponds to the pixel gray value, thus forming a width is 256 and the height is same as the original image, they consistent with the new image: V-disparity maps [13].

\subsection{Improved Linear Fitting Based on Hough Detection Method}

The image binarization is necessary in the first place, then edge detection and Hough transform and straight line fitting for obstacle detection need further. In this paper, through extracting line endpoint and the angle information of detected results, combining similar line to get a new line and the output line of linear Equation [14].Specific algorithm steps are as follows:

\section{The Improved Linear Fitting Based on Hough Detection Algorithm}

\section{STEPS}

- On the V-disparity map basic (after binarization processing) for canny edge detection

- According to the size of the image size decided Hough transform accumulator and allocate memory for it

- The Hough transform to the image, result will be put in the Hough transform accumulator. Set threshold, and according to the size threshold to take Hough transform in the accumulator accumulative value less than the threshold out, which is to think these points in the image is not a straight line

- Find the Hough transform to accumulate points of maximum value of accumulator, record this point, and take its neighborhood out. Continue to repeat lookup, until all accumulated value in the accumulator is zero. Recording of these points in the images of the corresponding linear

- According to the detected point draw a straight line in the image area, median filter to remove noise and enhance contrast, it follows binarization figure that containing only roads and barriers information 
- According to the line angle is close to the vertical get judgment on behalf of the border line (not close to the vertical), or on behalf of the obstacle line (vertical) approximation. The obstacle line segment group clustering get different obstacles, and finally with the least squares fitting line in each group

\section{The Experimental Results and Analysis}

In order to verify the feasibility of the algorithm in this paper, using a binocular camera actually collected path that contains obstacles of left and right images. As shown in Figure 4, the obstacles are clear. No camera calibration of stereo image of polar correction methods for correction. As shown in Figure 5.

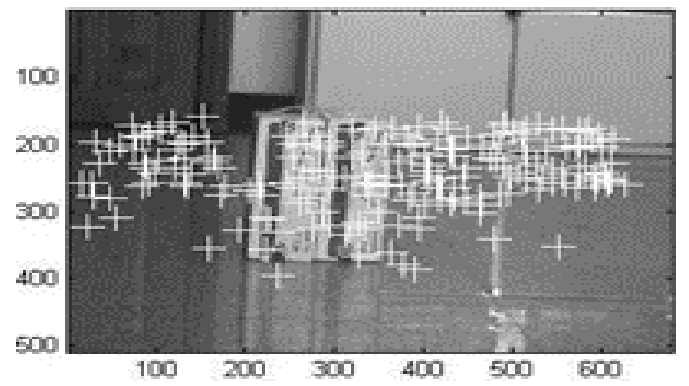

(a)

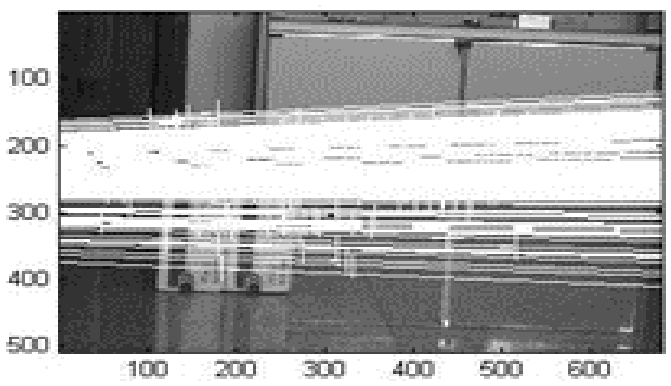

(b)

Figure 4. The Original Stereo Images: (a) The Left Image; (b) The Right Image

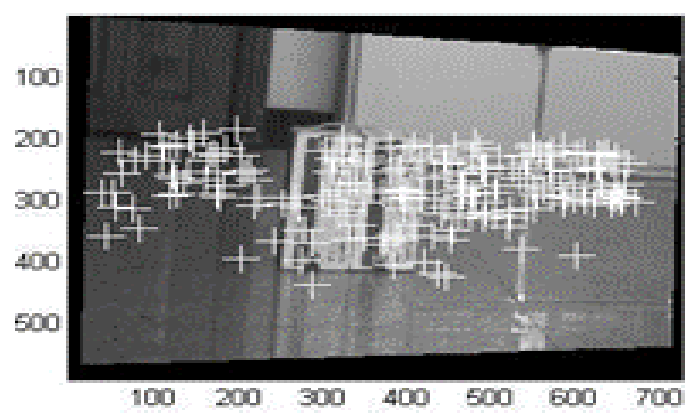

(a)

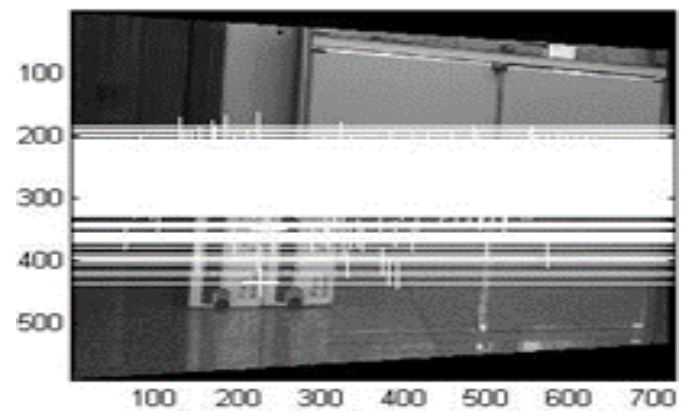

(b)

Figure 5. Polar Adjusted Stereo Image Pairs: (a) The Left Image; (b) The Right Image

After correction of the stereo image pairs for feature point tracking, it can be seen the two-dimensional image satisfying on the constraints of polar after correction, namely the polar and horizontal scan lines meet parallel relationship.

According to the two-dimensional image adjusted of the picture on the right selected as a benchmark figure to calculate dense disparity map, and to calculate the corresponding V-disparity map. As shown below: 


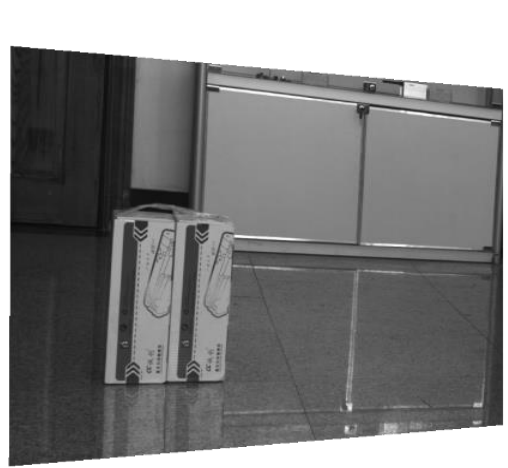

(a)

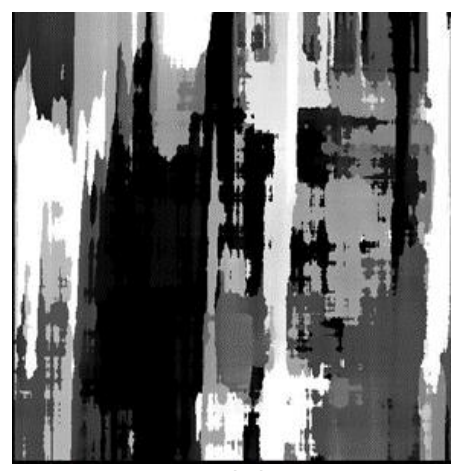

(b)

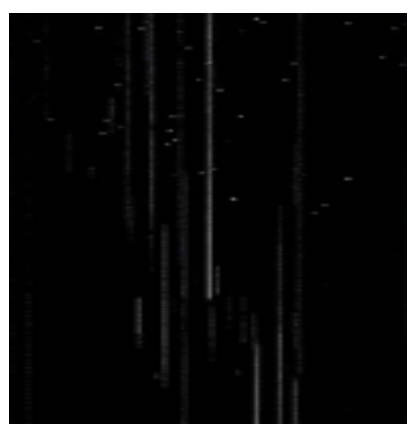

(c)

Figure 6. The Actual Scene Graph of V-disparity Map Calculation Process: (a) Right Image after the Correction; (b) Dense Disparity Map; (c) V-disparity Map

What can be seen from the V-disparity map is each a white line represents a barrier, a few more highlights of the white lines are the actual image of obstacles (carton) location. Because of the cartons are two independent individuals that be pasted together, Vdisparity map should not only get a straight line. Despite the presence of noise, the obstacles of general location is relatively easy to identify.

Figure 7 is to detect the straight lines in the V-disparity map, using the improved algorithm in this article compared with the traditional Hough detection algorithm. The results as follows:

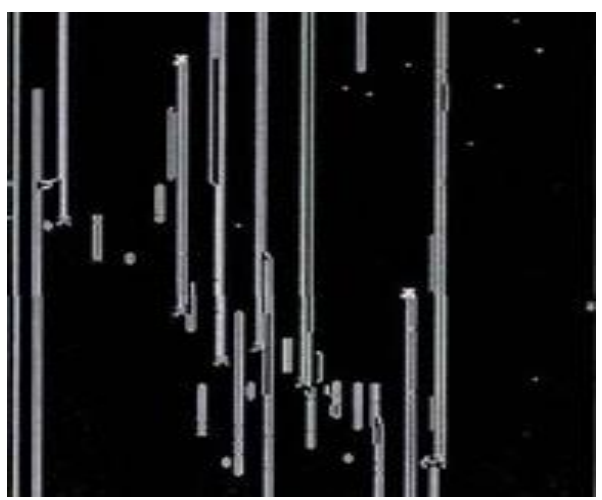

(a)

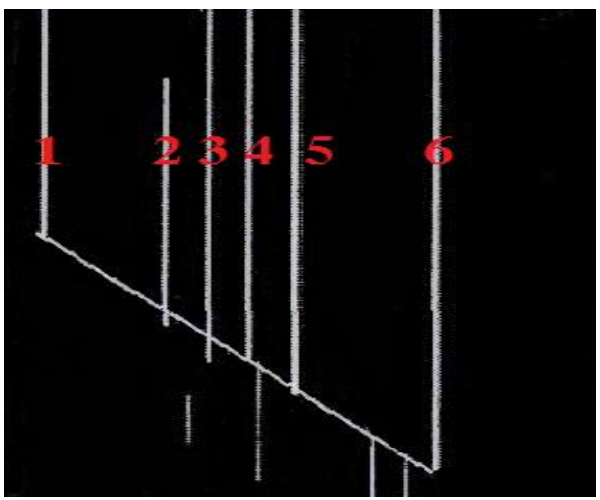

(b)

Figure 7. Straight Line Test Results Contrast: (a) The Traditional Hough Method to Detect Lines; (b) The Improved Algorithm to Detect Straight Lines

By comparing traditional Hough method to detect linear with the improved a linear fitting based on Hough detection method to detect linear in this paper, results can be seen that the effect of the latter is better than the former. Although the complexity of this algorithm is higher, the actual operation efficiency is relatively high and total elapsed time is $0.058 \mathrm{~s}$. Detecting obstacles can completely satisfy the real-time requirements, and has a strong anti-interference ability. It also has certain reference value to engineering application. Many times to collect images in the table below for testing line to compare the miss rate. 


\section{Table 2. Improved Algorithm Miss Rate Compared with the Traditional Method}

\begin{tabular}{|c|c|}
\hline $\begin{array}{c}\text { The Improved Algorithm } \\
\text { Miss Rate/\% }\end{array}$ & $\begin{array}{c}\text { Traditional Algorithm Miss } \\
\text { Rate/\% }\end{array}$ \\
\hline 12.78 & 20.91 \\
13.56 & 21.53 \\
12.82 & 24.62 \\
16.39 & 30.21 \\
13.74 & 26.28 \\
\hline
\end{tabular}

To the actual collection images containing obstacles on the path goes through a series of processing and uses V-disparity method to detect obstacles [15], etc. Eventually gets as shown in the following figure:

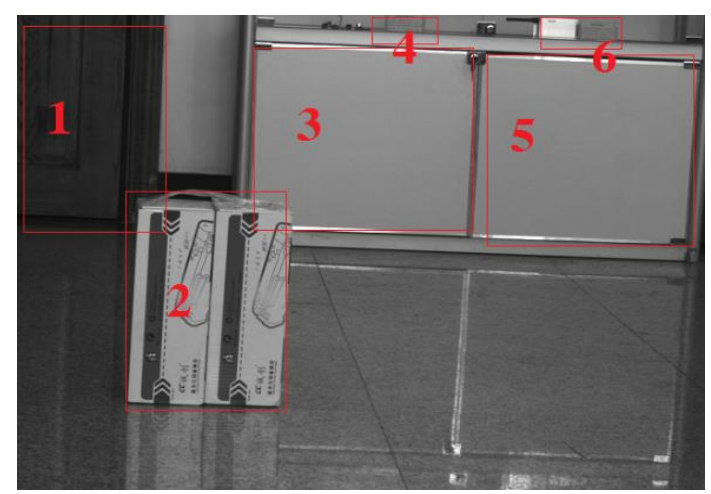

Figure 8. The Actual Environment Obstacle Detection Results

Among them, the obstacles 1: $4.5 \mathrm{~m}, 2: 2.3 \mathrm{~m}, 3: 4.2 \mathrm{~m}, 4: 4.23 \mathrm{~m}, 5: 4.6 \mathrm{~m}, 6$ : $4.67 \mathrm{~m}$.

It shows that this algorithm accurate detect obstacles, the distance and obstacles can also through the camera focal length and baseline distance calculated precision, label corresponds to the actual barrier.

We will get the distance information of binocular vision and sonar range information input to the T-S fuzzy neural network. The input is 5 sonar range value and the binocular distance measurement, after the offline training output robot control decisions so that the AS-R robot can avoid obstacles independently.

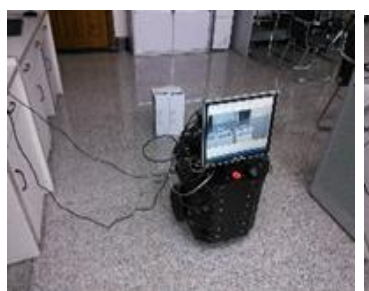

(a)

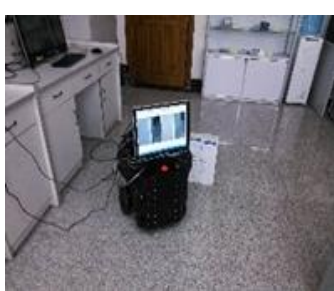

(b)

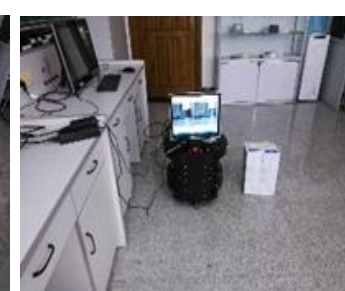

(c)

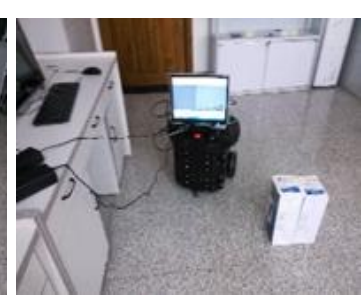

(d)

Figure 9. The Robot Avoid Obstacles Video Capture: (a)The Fifth Frame; (b)The Twenty-third Frame; (c)The Forty-second Frame; (d)The Fifty-sixth Frame

It can be seen that the robot can be safely around obstacles to reach target without touch, can meet the requirements of real-time obstacle avoidance, and has good stability. It proves that this algorithm is feasible. 


\section{Conclusions}

Based on binocular stereo vision matching technology, barrier extraction and other key problems, such as obstacle avoidance strategy method has carried on the thorough research and discussion, and carried out based on the binocular vision and sonar information fusion method of T-S fuzzy neural network control of mobile robot to avoid obstacles. The visual system can accurately detect the obstacles and distance of barriers, sonar sensors can pinpoint obstacles. Both mutual fusion improves the accuracy and is feasible under certain speed. However, this article only the indoor environment of the actual verification. In view of the outdoor environment is complicated, traffic information is more complex and barrier characteristics of diversity, it is difficult to guarantee this algorithm also has high adaptability. Therefore, subject to focus on research to solve in the future.

\section{Acknowledgements}

This work is partly supported by China Postdoctoral Science Foundation Funded Project (Grant No.2013M530954), the State Key Laboratory of Robotics Foundation (Grant No. 2012017), Program for Liaoning Excellent Talents in University (Grant No.LJQ2014021), the Natural Science Foundation of Liaoning Province (Grant No.2014020093), and Shenyang Ligong University Computer Application Key Discipline Foundation (Grant No.4771004kfx09). The authors also gratefully acknowledge the helpful comments and suggestions of the reviewers, which have improved the presentation.

\section{References}

[1] S. H. Zhang and W. Xiong, "Obstacle avoidance of the mobile robot based on binocular vision research", Journal of modern electronic technology, vol. 9, (2012), pp. 89-91.

[2] J. Yang, H. Sun and L. Wu, "Fuzzy neural network information fusion method in the application of robot obstacle avoidance", Automation technology and applications. vol. 2, (2005), pp. 31-33.

[3] Y. He and Y. F. Zhang, "Mobile robot obstacle avoidance algorithm based on binocular vision simulation", Computer simulation, vol. 2, (2013), pp. 282-285.

[4] Y. H. Xu, C. W. Zhang and H. Q. Xu, "Based on the laser range finder a new method of mobile robot obstacle avoidance", Robot, vol. 2, (2010), pp. 179-183.

[5] D. Wang, "Based on ultrasonic distance measurement with the fuzzy control of autonomous mobile robot obstacle avoidance", Ocean University of China, (2008).

[6] R. G. Luo and D. W. Tu, "Grid method of visual sensor integration and robot real-time obstacle avoidance", Computer engineering and application, vol. 24, (2011), pp. 233-235.

[7] H. Tao, H. S. Sawhney and R. Kumar, "A global matching framework for stereo computation", Proceedings of the 8th International Conference on Computer Vision(ICCV), (2001).

[8] X. Y. Qi, "V-disparity method based on binocular stereo vision obstacle detection algorithm research and implementation", China, Northeastern university, (2010).

[9] F. Z. Ceng and S. S. Bao, "A stereo matching algorithm of adaptive multiple window", Journal of computer science, vol. 6, (2012), pp. 519-521.

[10] H. L. Li, X. M. Li and L. J. Su, "Type based on T-S fuzzy neural network of wheeled robot obstacle avoidance method research", Computer measurement and control, vol. 1, (2011), 173-175.

[11] T. Fusiello, "A compact algorithm for rectification of stereo pairs", Machine Vision and Applications, vol. 1, (2000), pp. 16-22.

[12] Z. Hu and K. Uchimura, "U-v-disparity:an efficient algorithm for stereovision based scene analysis", Proceedings IEEE Intelligent Vehicles Symposium, (2005).

[13] R. Labayrade, D. Aubert and J. P. Tarel, "Real time obstacle detection in stereovision on non flat road geometry through "v-disparity" representation", IJCV, vol. 12, (2006), pp. 231-240.

[14] Q. Q. Dong, "The obstacle detection algorithm based on binocular stereo vision research and implementation", China, Northeastern university, (2009).

[15] Y. Li, "Obstacle recognition based on binocular vision research", Wuhan University of Technology, (2007). 


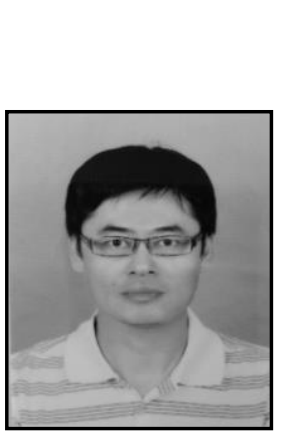

\begin{abstract}
Authors
Hongwei Gao, he received his Ph.D. degree in the field of pattern recognition and intelligent system from Shenyang Institute of Automation (SIA), Chinese Academy of Sciences (CAS) in 2007. Since September 2009, he has been an associate professor of information science \& engineering college, Shenyang Ligong University. Currently, he is the leader of academic direction for optical and electrical measuring technology and system. His research interests include digital image processing and analysis, stereo vision and intelligent computation. He has published more than sixty technical papers in these areas as first authors or co-authors.
\end{abstract}

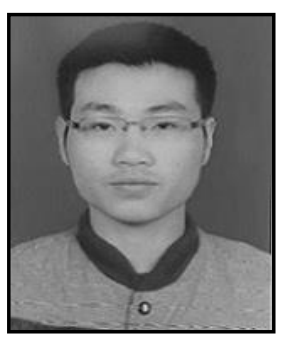

Qiuyang Wei, he Born in 1990 in Jiangsu Province, and received the B.S degree in Huaiyin Institute of Technology, China. Currently, he is pursuing the M.S degree in Shenyang Ligong University. The main research area is the Image Processing and Computer Vision.

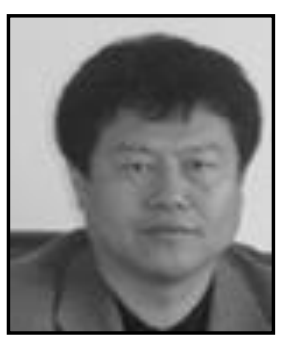

Yang $Y \mathbf{u}$, he received the B.S. degree in Control theory and Application from Shenyang Ligong University in 1990. Since 2003, he has been a Full Professor. Currently, he is the Associate Dean of School of Information Science \& Engineering, Shenyang Ligong University. His research interests include automatic detection and control, industrial process fault diagnosis. He has published more than forty technical papers in these areas.

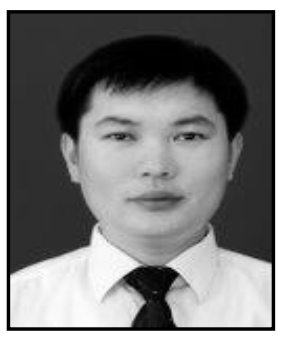

Jinguo Liu, he received his $\mathrm{Ph} . \mathrm{D}$. degrees in robotics from Shenyang Institute of Automation(SIA), Chinese Academy of Sciences(CAS) in 2007. Since January 2011, he has been a Full Professor with SIA, CAS. He also holds the Assistant Director position of State Key Laboratory of Robotics (China) from March 2008. His research interests include modular robot, rescue robot, space robot, and bio-inspired robot. He has authored and coauthored about sixty papers and twenty patents in above areas. 